\title{
Quasi-elastic neutron scattering in the multi-GPa range and its application to liquid water
}

\author{
S. Klotz, ${ }^{1, a)}$ Th. Strässle, ${ }^{2}$ and L. E. Bove ${ }^{1,3}$ \\ ${ }^{1}$ IMPMC, Université P\&M Curie, Case 115, 4 Place Jussieu, 75252 Paris, France \\ ${ }^{2}$ Paul Scherrer Institut, CH-5232 Villigen PSI, Switzerland \\ ${ }^{3}$ École Polytechnique Fédérale de Lausanne, Institute of Condensed Matter Physics, EPSL, CH-1015 \\ Lausanne, Switzerland
}

(Received 19 July 2013; accepted 18 October 2013; published online 4 November 2013)

\begin{abstract}
We present a method which allows quasi-elastic neutron scattering (QENS) at multi-GPa pressures, i.e., pressure conditions which are an order of magnitude higher than possible with QENS till now. The technique uses an opposed-anvil geometry with a sample chamber in the form of a toroid which minimizes multiple scattering effects. This method has been applied to measurements of the self-diffusion coefficient of water at $400 \mathrm{~K}$ up to $3 \mathrm{GPa}$. The results demonstrate the potential of this method for studying diffusion over a large pressure and temperature range. (C) 2013 AIP Publishing LLC. [http://dx.doi.org/10.1063/1.4828661]
\end{abstract}

Quasi-elastic neutron scattering (QENS) exploits the incoherent scattering cross section of certain elements-in particular hydrogen-to probe the stochastic (random) motion of atoms and molecules in liquids and solids. ${ }^{1}$ It is nowadays a well established technique and applied by a wide user community which spans material science to bioand soft condensed matter research. Typical applications are diffusion of hydrogen-rich liquids, the diffusion of hydrogen in metals, the study of molecular "rattling" in clathrates and framework structures, as well as the study of hydration water dynamics in biological matter. High pressure is a priori a highly sensitive parameter in such measurements. In simple liquids, for example, the diffusion coefficients are expected to decrease approximately exponentially with pressure, an argument based on the Stokes-Einstein relation and the observation that the viscosity is known to show the inverse pressure dependence. A survey of published high pressure QENS investigations reveals, however, that the current pressure limit is only approximately $0.6 \mathrm{GPa}^{2}$ The reason for this is partially related to the exceptionally strong incoherent cross section of hydrogen (80 barns) which leads to an attenuation length of typically $0.2 \mathrm{~mm}$. In such a case, the preferred sample holders are in the form of thin slabs, a geometry which is highly unfavorable for traditional high pressure cells.

Here we present a high pressure setup which allows QENS measurements to be carried out in the multi GPa range, i.e., at pressures which are an order of magnitude higher than possible so far. The technique (Figure 1) is based on an opposed-anvil geometry where-contrary to the traditional toroidal anvil method ${ }^{3}$ - the sample is not located in the center of the anvil culet but inside the toroidal gasket itself. The gasket consists of two symmetric parts with a groove of $0.5 \mathrm{~mm}$ depth and $19 \mathrm{~mm}$ diameter, which form a toroidal pressure chamber of $35 \mathrm{~mm}^{3}$ volume, once the two parts are put together. The choice of the gasket material was dictated by the requirement of metals with high strength and low incoherent and absorption cross sections. Copper

\footnotetext{
${ }^{\text {a) }}$ Author to whom correspondence should be addressed. Electronic mail: Stefan.Klotz@impmc.upmc.fr
}

beryllium hardened to $1000 \mathrm{MPa}$ yield strength and $10 \%$ elongation proved to be the best choice so far. Stainless steel gaskets made of an AISI 431 type alloy ("APX" from Aubert Duval) gave a more unfavorable signal/background ratio. The contact surface of the two parts was sputtered with $5 \mu \mathrm{m}$ titanium film to allow for cold welding under load. Anvils were made of cubic boron nitride $(\mathrm{cBN})$ sinter, the same material as used for diffraction experiments. ${ }^{4}$ They have a central bore of $6 \mathrm{~mm}$ diameter in which two $80 \mathrm{~W}$ cartridge heaters were tightly located. Careful shielding was achieved by sputtering gadolinium onto the conical faces of the anvils and using cadmium foil to cover parts of the cell which are close to the neutron beam. Temperatures were measured by two K-type thermocouples attached to each anvil, at approximately $1 \mathrm{~mm}$ distance from the gasket. These served also to control the output of two power supplies which fed the corresponding cartridge heaters. Calculations show that, due to the high thermal conductivity of $\mathrm{cBN}$, the temperature gradient between the sample position and the edge of the gasket is virtually zero. The forces needed to deform the gasket assembly and generate pressures in the multi-GPa range are typically 80 tonnes and were generated by a VX Paris-Edinburgh press which has a capacity of 130 tonnes. $^{4}$ The hydraulic pressure was provided by a manual oil pump and maintained at the desired values as the press slowly warmed up to $\sim 70{ }^{\circ} \mathrm{C}$ over several days of data collection.

To illustrate this technique, we show here data obtained on hot, dense liquid water at $400 \mathrm{~K}$ in the 0-3 GPa range (Figure 2). The measurements were carried out at the timeof-flight spectrometer FOCUS of the Swiss neutron source SINQ at the Paul Scherrer Institut using a wavelength of 5.0 $\AA$ (Ref. 5) and an instrumental resolution of $0.107 \mathrm{meV}$. FOCUS is a medium-intensity instrument and one might expect that high pressure QENS investigations on $35 \mathrm{~mm}^{3}$ samples are extremely difficult. The data shown in Fig. 2 prove the contrary, as long as the aim is limited to measuring translational diffusion coefficients which can be directly derived from the measured width of the QENS signal as a function of wave vector. Pressures were determined from the load on the anvils and linear interpolation between two fixed 


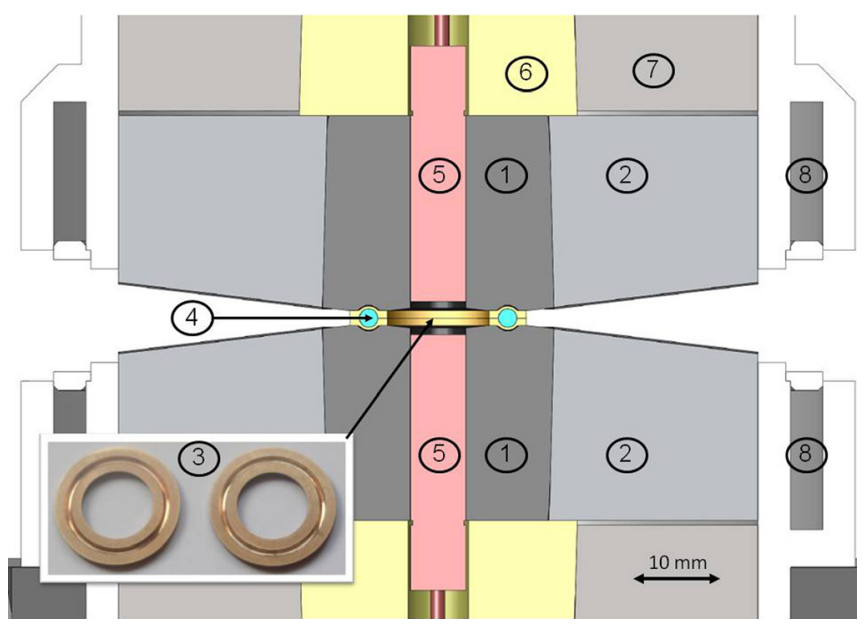

FIG. 1. Gasket-anvil assembly for high pressure QENS measurements. (1) and (2) anvil made of sintered cBN die supported by steel binding ring; (3) split $\mathrm{CuBe}$ gasket; (4) high pressure sample chamber; (5) cartridge heater; (6) and (7) anvil backing seat made of zirconia die and steel supporting ring; (8) cooling rings. Conical anvil faces (thick lines) are covered with gadolinium film.

points where the pressure is accurately known: $0 \mathrm{GPa}(13$ tonnes) and the pressure where water at $400 \mathrm{~K}$ crystallizes into ice VII, i.e., $3.0 \mathrm{GPa}$ (70 tonnes) ${ }^{6}{ }^{\text {Crystallization is eas- }}$ ily observable by the suppression of the quasi-elastic signal in the measured spectra.

Figure 2 shows QENS spectra at a (average) wave vector $\mathrm{Q}=0.82 \AA^{-1}$, at low and high pressure, after $9 \mathrm{~h}$ of

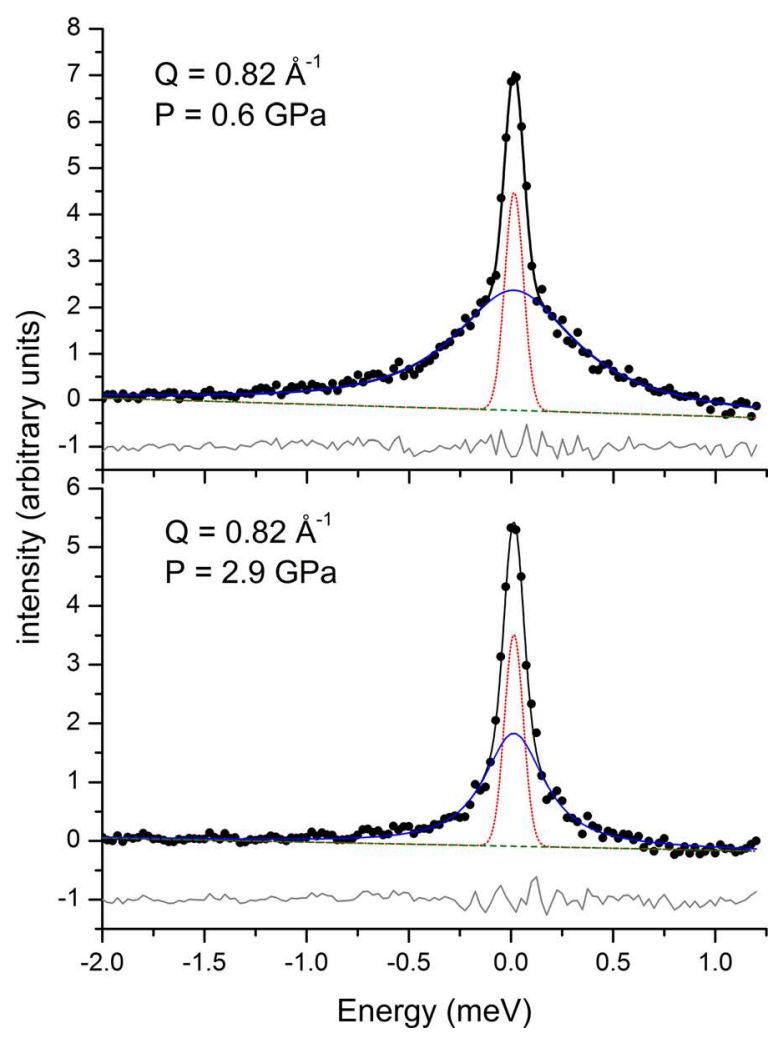

FIG. 2. QENS spectra of liquid water at low and high pressure (0.6 and 2.9 $\mathrm{GPa}$ ), at an intermediate Q-value of $0.82 \AA^{-1}$. The fits through the measured data points (solid circles) are shown by the black lines; the difference curves are given below. The elastic contributions are indicated by the dotted (red) lines, the linear background by the dashed (green) lines. The pressure effect on the Lorentzian QENS line widths within the Pseudo-Voigt (solid blue line) is easily visible. accumulation time. We consider these as representative for several dozen of spectra covering a $\mathrm{Q}$ range between 0.35 and $1.4 \AA^{-1}$ and 6 pressure points. As the gap between the anvils closes with increasing load (pressure) the measured signal is seen to decrease by $\sim 25 \%$, while the ratio between quasielastic and elastic contributions remains approximately constant. After data reduction using standard software (DAVE) and binning in 10 Q-groups, the QENS spectra were fitted to three contributions ("model (1)"): (a) a Gaussian with fixed width given by the instrumental resolution which describes the elastic scattering coming essentially from the gasket; (b) a Pseudo-Voigt resulting from the quasielastic signal of the sample with its Gaussian width fixed to the instrumental resolution and an adjustable parameter for its Lorentzian width; (c) a linear background with adjustable slope. To derive translational diffusion constants, a simple continuous diffusion model was used which predicts a $Q^{2}$ dependence of the Lorentzian line width as $\mathrm{Q} \rightarrow 0$. To investigate the possible correlation between the fitted QENS line-width and the way the background was modeled, an analysis was carried out with a background determined from the same sample which was crystallized into ice VII (model (2)). Using this model, a second independent analysis was carried out with the fitted energy window increased to $-7 \mathrm{meV}$ to $+1.25 \mathrm{meV}$ (model (3)). Figure 3 shows line width for the lowest and highest pressure points determined by model (1) which gives a linear dependence in $Q^{2}$ but a slight non-zero intercept of approximate $50 \mu \mathrm{eV}$, i.e., $50 \%$ of the instrumental resolution. To investigate the source of this deficiency, multiple-scattering corrections were carried out, again for the lowest and highest pressure points. For this purpose multiple-scattering occurring within both the sample and the gasket was modeled using the well-assessed procedure previously employed for inelastic neutron scattering (INS) experiments with low transmission samples. ${ }^{7}$ The Monte Carlo program of Ref. 8 was adapted in order to generate random trajectories to the special toroidal geometry of the sample/gasket assembly. The contribution from the

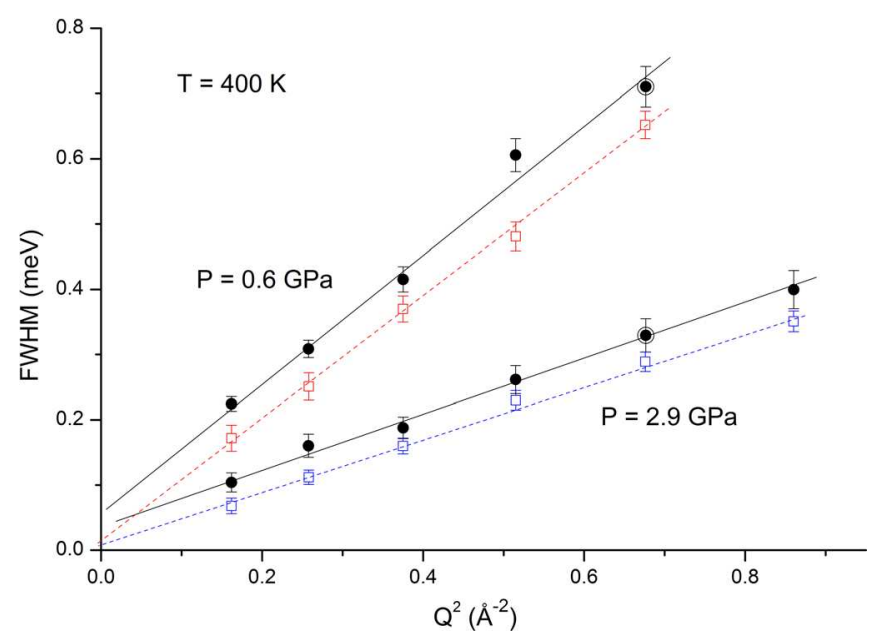

FIG. 3. Full width at half maximum (FWHM) of the fitted QENS signal plotted as a function of $\mathrm{Q}^{2}$, at $0.6 \mathrm{GPa}$ and $2.9 \mathrm{GPa}$. The marked dots $(\odot)$ at $0.86 \AA^{-2}$ are those shown in Figure 2. Full symbols correspond to analysis using background model (1), open symbols include multiple scattering corrections, as explained in the text. The slopes correspond to diffusion constants as shown in Figure 4, derived via the relation $\mathrm{D}=0.1519 \times 10^{-3} \times$ (FWHM/2)/Q ${ }^{2}$, where D is in $\mathrm{cm}^{2} / \mathrm{s}$, FWHM in $\mathrm{meV}$, and $\mathrm{Q}$ in $\AA^{-1}$. 


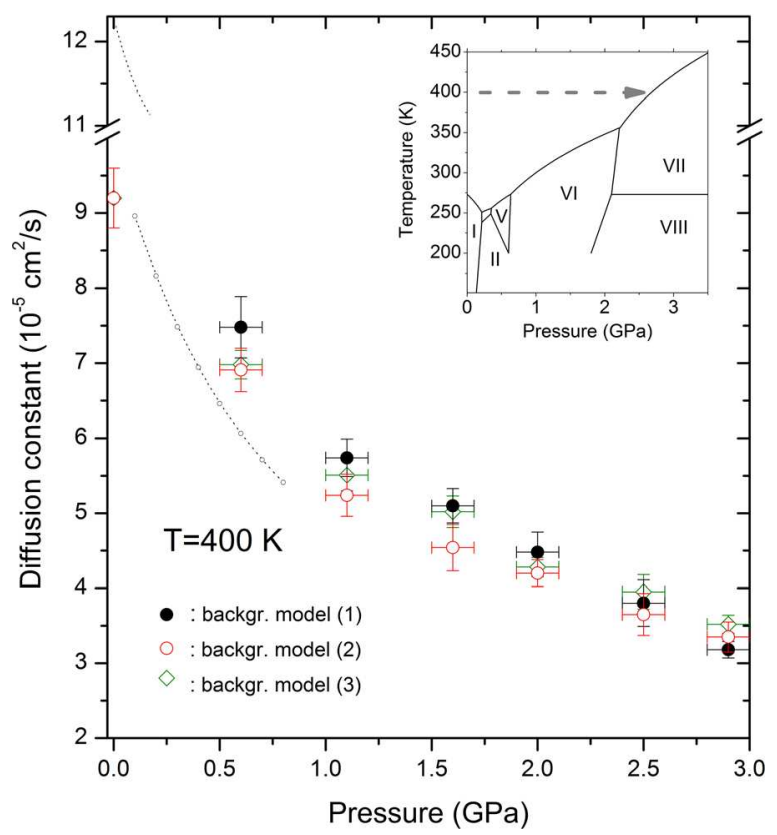

FIG. 4. Pressure dependence of diffusion coefficients of liquid water at $400 \mathrm{~K}$. Different symbols indicate different methods of evaluating the background, see text for details. Dashed lines are NMR results to 0.17 GPa (Ref. 9) and $0.8 \mathrm{GPa}$ (Ref. 10). Inset: Phase diagram of water with P-T path of measurements shown by an arrow.

pressure cell was measured by collecting inelastic scans on the empty gasket, and the procedure was checked experimentally by measuring the total scattering from samples with different thicknesses. Figure 3 demonstrates that the data obtained by this procedure give an intercept which is now virtually zero without changing significantly the slope, hence the diffusion coefficients. Figure 4 shows that the different ways to analyze the data have a minor influence on the values derived for the diffusion constants, and gives an estimate of the effect of the model choice on the numerical value derived for diffusion coefficient from the present data.

In principle, QENS is also able to detect rotational (i.e., local) motion and determine corresponding diffusion coefficients (or relaxation times). The signal from rotational diffusion manifests in a broad and relatively weak signal which is difficult to differentiate from the background signal if the counting statistics is limited, as it was the case in our study. With better statistics available, a somewhat larger energy window, and multiple scattering corrections, measurements of rotational diffusion are feasible using the same pressure set-up. ${ }^{15}$

The pressure dependence of the diffusion coefficient reported here can be compared with published results from nuclear magnetic resonance (NMR) measurements under pressure, i.e., the data of Krynicki et al. on $\mathrm{H}_{2} \mathrm{O}$ to $0.17 \mathrm{GPa}$ (Ref. 9) and Wilbur et al. on heavy water to $0.8 \mathrm{GPa},{ }^{10}$ see lines in Fig. 4. For this purpose, the reported temperature dependence was interpolated to $400 \mathrm{~K}$ using an Arrhenius law, the most unbiased approach given the scattering of data of Wilbur et al. when plotted on an isobar. The data on $\mathrm{D}_{2} \mathrm{O}$ were scaled by a factor sqrt(20/18) to get approximate values of $\mathrm{H}_{2} \mathrm{O}$. It is seen in Fig. 4 that the absolute values of the two NMR data differ by $\sim 20 \%$ with the neutron data falling between them, and the pressure dependence is reasonable consistent with the NMR results.

Our measurements indicate a self diffusion constant of water at the melting line at $400 \mathrm{~K}$ and $2.9 \mathrm{GPa}$ of $3.1 \times 10^{-5} \mathrm{~cm}^{2} / \mathrm{s}$ which is approximately 3 times larger than at the melting line at ambient pressure $\left(1.1 \times 10^{-5} \mathrm{~cm}^{2} / \mathrm{s}\right.$, (Ref. 10)). The effect of pressure is hence to compensate partially the strong effect of temperature to give diffusion coefficients which seem to vary little along the melting line, a finding which recalls the behavior of the shear viscosity along the melting line. ${ }^{11}$

The possibility of QENS measurements in the multiGPa range will allow systematic studies of the self-dynamics of hydrogen-rich fluids across large density variations, in particular pure hydrogen where the volume reduction between the liquid and the melting point at $5.5 \mathrm{GPa}$ at $300 \mathrm{~K}$ is $\sim 300 \%$. Moreover, the study of relaxational dynamics in hydrogen-rich glass forming systems under significant variation of density will allow disentangling the effects of the reduced volume, enhancing phenomena such as molecular hopping, from those linked to the dynamical arrest observed by approaching the glass transition temperature.

The method will be highly interesting also for solids which have low compressibility and where pressure effects on diffusion constants have so far been impossible to detect using classical gas and piston-cylinder devices. A typical example is hydrogen diffusion in metals where pressures in the kbar range lead to volume changes of typically only $0.1 \%$. Such density changes are too small to have measurable effects on diffusion constants, as shown in data on $\mathrm{NbH}_{\mathrm{x}}$, for example. ${ }^{12}$ Pressures in the GPa range would allow testing predictions of various microscopic models ${ }^{13}$ of $\mathrm{H}$-diffusion in metals which have been proposed since the early 1980s but remained so far experimentally inaccessible.

Finally, the possibility to probe the diffusion in simple hydrogen-based molecular systems in the GPa range will provide a precise determination of the diffusive processes in geophysically relevant conditions. Water diffusion in porous rocks under a few $\mathrm{GPa}$ is relevant to understanding water mobility in the transition zone of the upper mantle. Methane diffusion in high pressure clathrate phases could be relevant to understand the anomalous methane releases registered on Mars, ${ }^{14}$ and knowledge of proton diffusion at high pressures and high temperatures in water or ammonia ices would allow to better characterize the geodynamics of icy bodies in the universe.

We wish to thank J. Philippe (IMPMC) for help in the design of the system and preparation of the experiments, as well as M. Zolliker (PSI) and M. Bartkowiak (PSI) for technical assistance during the measurements. This work is based on experiments performed in 2007 and 2008 at the Swiss spallation neutron source SINQ, Paul Scherrer Institute, Villigen, Switzerland and was financially supported by the French Agence Nationale de la Recherche (ANR JCJC0135).

\footnotetext{
${ }^{1}$ M. Bée, Quasielastic Neutron Scattering (Taylor \& Francis, London, 1988).

${ }^{2}$ S. Klotz, Techniques in High Pressure Neutron Scattering (CRC-Taylor \& Francis, Boca Raton, 2013).
} 
${ }^{3}$ L. G. Khvostantsev, L. F. Vereshchagin, and A. P. Novikov, High Temp. High Press. 9, 637 (1977).

${ }^{4}$ S. Klotz, Th. Strässle, G. Rousse, G. Hamel, and V. Pomjakushin, Appl. Phys. Lett. 86, 031917 (2005).

${ }^{5}$ S. Janssen, J. Mesot, L. Holitzer, A. Furrer, and R. Hempelmann, Physica B 234-236, 1174 (1997).

${ }^{6}$ C. W. F. T. Pistorius, E. Rapoport, and J. B. Clark, J. Chem. Phys. 48, 5509 (1968).

${ }^{7}$ L. E. Bove, B. Dorner, C. Petrillo, F. Sacchetti, and J. B. Suck, Phys. Rev. B 68, 024208 (2003); L. E. Bove, F. Formisano, F. Sacchetti, C. Petrillo, A. Ivanov, B. Dorner, and F. Barocchi, Phys. Rev. B 71, 014207 (2005).

${ }^{8}$ C. Petrillo and F. Sacchetti, Acta Crystallogr., Sect. A: Found. Crystallogr. 46, 440 (1990).
${ }^{9}$ K. Krynicki, Ch. D. Green, and D. W. Sawyer, Faraday Discuss. 66, 199 (1978).

${ }^{10}$ D. J. Wilbur, T. DeFries, and J. Jonas, J. Chem. Phys. 65, 1783 (1976).

${ }^{11}$ E. H. Abramson, Phys. Rev. E 76, 051203 (2007).

${ }^{12}$ U. Stuhr, H. Wipf, C. Vettier, and H. R. Schober, Solid State Commun. 80, 987 (1991).

${ }^{13}$ O. J. Zogal and R. M. Cotts, Phys. Rev. B 11, 2443 (1975); D. Steinbinder, H. Wipf, H. R. Schober, H. Blank, G. Kearley, C. Vettier, and A. Magerl, Europhys. Lett. 8, 269 (1989).

${ }^{14}$ O. Mousis, E. Chassefière, J. Lasue, V. Chevrier, M. E. Elwood Madden, A. Lakhlifi, J. I. Lunine, F. Montmessin, S. Picaud, F. Schmidt et al., Space Sci. Rev. 174, 213 (2013).

${ }^{15}$ L. E. Bove, S. Klotz, Th. Strässle, M. Koza, J. Teixeira, and A. M. Saitta, Phys. Rev. Lett. 111, 185901 (2013). 